\title{
Adult Patients with Eosinophilic Esophagitis Do Not Show an Increased Frequency of the HLA-DQ2/DQ8 Genotypes Predisposing to Celiac Disease
}

\author{
Alfredo J. Lucendo • Ángel Arias • Isabel Pérez-Martínez • \\ Antonio López-Vázquez · Jesús Ontañón-Rodríguez • \\ Sonia González-Castillo $\cdot$ Livia C. De Rezende $\cdot$ Luis Rodrigo
}

Received: 1 June 2010/Accepted: 29 July 2010/Published online: 20 August 2010

(C) Springer Science+Business Media, LLC 2010

\begin{abstract}
Background Recent articles have described patients that share eosinophilic esophagitis (EoE) and celiac disease (CD) suggesting a true relationship between both diseases. Aims The purpose of this study was to investigate whether HLA DQ2 and DQ8 predisposing to CD are increased in adult patients with EoE.

Methods HLA alleles conferring risk for CD was assessed in 75 adult EoE patients attended at two hospitals located in different Spanish regions over the past 2 years. We compared the frequencies to the registered data of 421 healthy kidney and bone marrow donors from our hospitals for the following alleles: (a) DR3-DQ2 haplotype; (b) the combination of DR3-DQ2 and DR4-DQ8; (c) DR4-DQ8 haplotype; (d) the simultaneous presence of the DR5-DQ7 and DR7-DQ2 haplotypes; and lastly (e) any combination
\end{abstract}

A. J. Lucendo $(\bowtie)$ · S. González-Castillo · L. C. De Rezende Department of Gastroenterology, Hospital General de

Tomelloso, Vereda de Socuéllamos, s/n,

13700 Tomelloso, Ciudad Real, Spain

e-mail: alucendo@vodafone.es

Á. Arias

Research Unit, Complejo Hospitalario La Mancha Centro,

Alcázar de San Juan, Spain

I. Pérez-Martínez $\cdot$ L. Rodrigo

Department of Gastroenterology, Hospital Central

de Asturias, Oviedo, Spain

\author{
A. López-Vázquez \\ Department of Immunology, Hospital Central \\ de Asturias, Oviedo, Spain \\ J. Ontañón-Rodríguez \\ Department of Immunology, Hospital General \\ Universitario de Albacete, Albacete, Spain
}

of haplotypes not conferring risk for the development of CD.

Results The HLA DQ2 and DQ8 alleles were analyzed in 58 adult EoE patients from hospital \#1 and in 20 patients from hospital \#2, and they were compared to recorded HLA genotyping data from 298 and 123 healthy donors, respectively. No differences were found between the distribution of the HLA frequencies of the patients and controls at both hospitals and the data could be combined. EoE patients did not show increased frequencies of DQ2 and DQ8 alleles compared to controls.

Conclusions Our work does not allow us to establish a common genetic basis for $\mathrm{EoE}$ and $\mathrm{CD}$ because an increased frequency of the HLA DQ2 and DQ8 alleles predisposing to $\mathrm{CD}$ was not observed in adult EoE patients compared to controls.

Keywords Eosinophilic esophagitis - Celiac disease . HLA antigens - DQ2 genotype - DQ8 genotype

\section{Introduction}

Celiac disease (CD) and eosinophilic esophagitis (EoE) are two different well-known diseases which affect the gastrointestinal mucosa. The former is located in the small bowel and the latter in the esophagus. Both diseases are predominantly diagnosed in young patients, but can appear at any stage of life.

EoE is an immunoallergic disease characterized by chronic, recurrent esophageal symptoms and dense esophageal eosinophilic infiltrate [1]. The pathogenesis of EoE includes a Th2-type immunological reaction, which is triggered by exposure to dietary allergens causing infiltration of the esophageal mucosa by $\mathrm{T}$ lymphocytes, mast 
cells and eosinophils [2]. The prevalence of EoE has risen over the last few decades to the same extent as other immunoallergic diseases.

$\mathrm{CD}$ is an autoimmune disease triggered by the ingestion of food containing gluten and manifests in genetically susceptible individuals, giving rise to different types of lesions in the small bowel mucosa [3, 4]. CD affects between 1 and $3 \%$ of the European and US population at some stage in life [5]. CD has a higher class II MHC association than detected previously in many other autoimmune diseases [6]. Approximately $90 \%$ of celiac patients carry the HLA-DQ2 heterodimer which is encoded by the DQA $1 * 05$ and DQB $1 * 02$ genes carried either in cis position on the DR3-DQ2 haplotype, which is common to many autoimmune diseases, or in trans, where the a chain is encoded on the DR5-DQ7 (DRB1*11/12, DQA $1 * 0505$, DQB1*0301) haplotype on one chromosome and the b chain on the DR7-DQ2 (DRB1*07, DQA1*0201, DQB1*0202) haplotype on the other chromosome [7]. Most patients who are DQ2 negative carry the DQ8 genotype (DRB1*04, DQA1*0301, DQB1*0302). DQ2 and DQ8 molecules present gluten peptides or related antigens to disease-specific CD4+ $\mathrm{T}$ cells.

The relationship between EoE and CD was recently brought to our attention and both disorders could have various aspects in common. A number of case reports have been published in relation to children displaying signs of both diseases [8-11] and a true association is suggested to exist between them.

However, so far, the relationship between EoE and CD has not been systematically studied. This article analyzes the presence of HLA DQ2 and HLA DQ8 conferring risk for $\mathrm{CD}$ in a large group of EoE patients at two Spanish hospitals. It compares the distribution of these alleles to the reference frequency observed in a wide group of healthy donors from our hospitals, representing the general Spanish population.

\section{Patients and Methods}

The presence of HLA alleles conferring risk for CD was assessed in all the adult EoE patients ( $\geq 16$ year old) who were attended at two general hospitals in different Spanish regions over the past 2 years (\#1, Hospital General de Tomelloso and \#2, Hospital Central de Asturias). EoE was diagnosed based on accepted criteria following the examination of esophageal, gastric and duodenal biopsies [1]. EoE was diagnosed by esophageal and/or upper gastrointestinal tract symptoms accompanied by $\geq 15$ intraepithelial eosinophils/HPF in 1 or more biopsy specimens without pathologic GERD, as shown by normal $\mathrm{pH}$ monitoring of the distal esophagus or the lack of response to high-dose proton pump inhibitor (PPI) medication. Gastric and duodenal biopsies were assessed in order to exclude eosinophilic gastroenteritis. Villous atrophy and lymphocytic infiltration were also excluded in duodenal samples.

Four different haplotypes for $\mathrm{CD}$ were analyzed in all patients using the polymerase chain reaction single nucleotide polymorphism techniques (PCR-SNPs) performed on peripheral blood leukocytes. We specifically assessed: (a) the presence of the DR3-DQ2 haplotype with no distinction between homozygosis or heterozygosis with another nonrisk allele; (b) the presence of the DR3-DQ2-DR4-DQ8 combination; (c) the expression of DR4-DQ8 haplotype (with no distinction between homozygosis or heterozygosis with another non-risk allele); (d) the simultaneous presence in trans combination of the DR5-DQ7 and DR7-DQ2 haplotypes; and (e) any combination not conferring risk for the development of $\mathrm{CD}$.

The estimated distribution of the alleles in the reference populations was obtained from the registered data of 421 unrelated healthy individuals compiled in two databases, of which 298 were kidney and bone marrow donors from the area of hospital \#1, and 123 were bone marrow donors from the area of hospital \#2. The geographical origin of the numerous control groups enabled them to represent the distribution of the alleles in each reference population.

We compared the relative frequencies (percentages) of the distribution of antigens between the patient and control groups using chi-square tests (Fisher's exact test, where appropriate). Statistical analyses were performed using statistical analysis software PASW 18.0 (SPSS Inc).

This research was carried out in accordance with the Helsinki Declaration and approved by the ethics committees in our institutions.

\section{Results}

HLA alleles predisposing to CD were analyzed in 58 adult EoE patients attended at hospital \#1 (50 males, 8 females; mean age 32.2 years, standard deviation [SD] 12.7) and in 20 at hospital \#2 (14 males, 6 females; mean age 36.55 years, SD 20.28) and were compared to registered HLA frequencies in the donor databases at each hospital, respectively.

We did not observe any increased frequency of the DQ2 and DQ8 alleles in the EoE patients at each hospital compared to the respective control groups (Table 1). In statistical terms, no significant differences were observed regarding each HLA haplotype in the respective control and patient groups at each hospital $(P>0.05)$, and the data could be combined.

Overall, there were no differences in the distribution of the proportions of each analyzed haplotype between EoE 
Table 1 Distribution and frequencies of the HLA DQ2 and DQ8 alleles conferring risk for celiac disease (CD) in two series of EoE patients at two Spanish hospitals compared to the frequencies of HLA
DQ2 and DQ8 in the control groups comprising healthy kidney and bone marrow donors at each hospital

\begin{tabular}{|c|c|c|c|c|c|c|c|c|c|c|}
\hline \multirow[t]{2}{*}{ CD-related HLA antigens } & \multicolumn{5}{|l|}{ Hospital \#1 } & \multicolumn{5}{|l|}{ Hospital \#2 } \\
\hline & $\begin{array}{l}\text { Number in } \\
\text { patient group }\end{array}$ & $\%$ & $\begin{array}{l}\text { Number in } \\
\text { control group }\end{array}$ & $\%$ & $P$ & $\begin{array}{l}\text { Number in } \\
\text { patient group }\end{array}$ & $\%$ & $\begin{array}{l}\text { Number in } \\
\text { control group }\end{array}$ & $\%$ & $P$ \\
\hline DR3 DQ2 & 10 & 17.24 & 76 & 25.5 & 0.18 & 5 & 25 & 28 & 22.7 & 0.78 \\
\hline DR3 DQ2/DR4 DQ8 & 3 & 5.17 & 6 & 2 & 0.17 & 1 & 5 & 4 & 3.2 & 0.54 \\
\hline DR4 DQ8 & 4 & 6.90 & 49 & 16.4 & 0.06 & 1 & 5 & 15 & 12.2 & 0.7 \\
\hline DR7 DQ2/DR5 DQ7 & 4 & 6.90 & 12 & 4 & 0.31 & 1 & 5 & 8 & 6.5 & 1 \\
\hline Haplotype not conferring risk & 37 & 63.79 & 155 & 52 & 0.1 & 12 & 60 & 68 & 55.3 & 0.69 \\
\hline Total & 58 & 100 & 298 & 100 & & 20 & 100 & 123 & 100 & \\
\hline
\end{tabular}

The DR4-DQ8 haplotype was more frequent in the control groups than in the patient groups, which gained statistical significance following the inclusion of patient series from both hospitals

patients and controls, except for the DR4-DQ8 haplotype, which was significantly increased in the control group compared to the EoE patients $(P=0.039)$. These data are shown in Table 2 and Fig. 1.

\section{Discussion}

Our work analyzes the frequency of the well-known classII MHC antigens predisposing to CD-HLA DQ2 and DQ8-in a series of adult EoE patients at two Spanish hospitals. Both centers have genetically homogenous patients as no differences in allele distribution were observed between them. Given that the frequencies of haplotypes predisposing to $\mathrm{CD}$ were not increased in $\mathrm{EoE}$ patients compared to the control groups, we are unable to establish a true association between both disorders.

Over the past few years, several studies have identified a potential relationship between both diseases based on certain clinical observations. In a case series of ten patients with EoE correlatively diagnosed in Australia, it was reported that eight out of them expressed the HLA-DQ2 haplotype (with a frequency affecting approximately $45 \%$ of the local population), and one more was DQ8+ [12]. In an Italian pediatric case series of $16 \mathrm{EoE}$ patients, six children were simultaneously diagnosed with $\mathrm{CD}$ based on

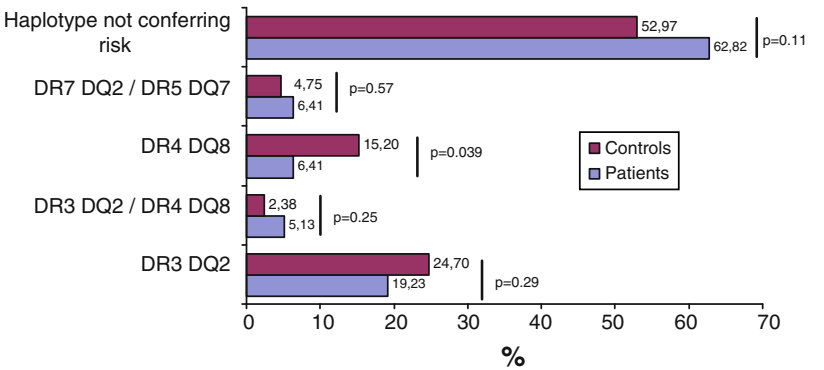

Fig. 1 Comparative representation of the frequencies of each HLA allele conferring risk for $\mathrm{CD}$ in our series of EoE patients and control groups from our study. No statistical differences were identified between the two study groups

serological criteria and duodenal villous atrophy [10]. Another Italian case report presented three children with both EoE and CD who showed favorable clinical and histological esophageal evolution after they followed glutenfree diets [9]. Lastly, a recently published study aimed to show the relationship between both diseases by estimating the prevalence of EoE among children diagnosed with $\mathrm{CD}$ at an Australian institution over an 8-year period. The authors discovered that at least $4 \%$ of the children with $\mathrm{CD}$ also suffered from EoE, and they suggested that this percentage could be higher if esophageal biopsies had been systematically sampled in each patient undergoing an
Table 2 Distribution and frequencies of the HLA DQ2 and DQ8 alleles in our series of EoE patients at two Spanish hospitals compared to the control groups

The proportion of the DR4 DQ8 haplotype was significantly higher in the control groups than the EoE patients $(P=0.039)$

\begin{tabular}{lcrrrl}
\hline HLA antigens & $\begin{array}{l}\text { Number } \\
\text { of patients }\end{array}$ & \multicolumn{1}{l}{$\%$} & $\begin{array}{l}\text { Number } \\
\text { of controls }\end{array}$ & \multicolumn{1}{l}{$\%$} & $P$ \\
\hline DR3 DQ2 & 15 & 19.23 & 104 & 24.70 & 0.29 \\
DR3 DQ2/DR4 DQ8 & 4 & 5.13 & 10 & 2.38 & 0.25 \\
DR4 DQ8 & 5 & 6.41 & 64 & 15.20 & 0.039 \\
DR7 DQ2/DR5 DQ7 & 5 & 6.41 & 20 & 4.75 & 0.57 \\
Haplotype not conferring risk & 49 & 62.82 & 223 & 52.97 & 0.11 \\
Total & 78 & 100 & 421 & 100 & \\
\hline
\end{tabular}


endoscopy for suspected CD [11]. In contrast to these findings, the majority of them referred to children; our study was unable to demonstrate a common genetic basis for EoE and CD in adults. A direct relationship between both diseases cannot be supported attending to the frequency of genetic susceptibility markers as opposed to in the case of certain autoimmune diseases [13], the prevalence of which is much higher in $\mathrm{CD}$, especially type-1 diabetes mellitus and autoimmune thyroid disorders [14].

Some reasons could have led some authors to consider the possibility of a real relationship between EoE and CD. Both are mainly chronic inflammatory digestive diseases determined by exposure to certain dietary components. Both EoE and CD are frequently associated with a wide variety of extra intestinal manifestations and clinically respond to the elimination of specific foods from the diet or to treatment using steroidal anti-inflammatory drugs. Both diseases clearly run in families. However, CD is a genetically determined disorder affecting individuals who majorly express the HLA DQ2 and DQ8 haplotypes, which are considered necessary but are not responsible for the development of the disease alone. This genetic basis determines a frequent association of cases within the same family, reaching up to $20 \%$ in first degree relatives and up to $75 \%$ in monozygotic siblings [15]. Many cases of EoE have also been described to run in families [16-19]. This would indicate that the disease has a relatively strong genetic background and no differences in clinical, pathological or molecular characteristics have been observed between sporadic and family-related cases of EoE [20]. Furthermore, given the high prevalence of CD in Europe [5], this condition might simply overlap with EoE as a coincidence. This would explain some of the cases in the literature where the two diseases do clearly co-exist.

Conversely, several epidemiological reasons distinguish $\mathrm{CD}$ from EoE, e.g. CD predominantly affects females at a ratio of 2:1 irrespective of race [15], and differences were observed in different geographical regions in accordance with dietary habits (i.e. more rice than wheat in the staple Asian diet). EoE predominates in males, with a 3:1 ratio to women, and has been mainly described in Caucasians [21, 22]. Although CD patients may present respiratory or skin symptoms [15], atopic manifestations are nearly always associated with EoE [23].

Our work shows that frequencies of risk alleles for CD are not increased in EoE adult patients compared to the control population. Consequently, we cannot establish a common genetic basis for both diseases because we lack sufficient evidence of HLA DQ2 and DQ8 predisposing to the development of EoE. We unexpectedly found a significantly higher frequency of the DR4-DQ8 haplotype in the control group compared to EoE patients $(P=0.039)$. Although the results were difficult to interpret, we assume that EoE patients express the DR4-DQ8 allele with a reduced frequency, which is only present in about $10 \%$ of celiac patients. This data, which must be confirmed by further studies, encourages us to carry out further research on the genetic markers and on the putative role of different antigen-presenting molecules in the development of EoE.

Furthermore, our results do not completely rule out the possible role of gluten exposure in a small number of EoE patients exhibiting HLA DQ2 and/or DQ8. Our study shows that these alleles are not present in EoE at a greater rate than in healthy controls, but it does not provide any detail into the reverse issue, i.e. if patients with celiac disease are more likely to have EoE, as suggested by a recent paper [11]. We should bear in mind that esophageal eosinophilic infiltration could be a gluten-associated manifestation, at least in a small subgroup of pediatric patients with $\mathrm{CD}$, and could be caused by CD itself. HLA DQ2 and DQ8 are also genetic risk factors for other diseases which have an autoimmune basis [13] and are much higher in CD [14]. There is evidence, although somewhat controversial, that if $\mathrm{CD}$ is left untreated it could predispose to the development of other autoimmune diseases, including Sjögren's syndrome, Addison's disease, autoimmune liver disease, cardiomyopathies and neurological disorders. In this respect, some cases of EoE (an inflammatory disorder of immunological origin) could be triggered by $\mathrm{CD}$, which has not yet been diagnosed. These matters require further research.

Acknowledgments We would like to thank Dr Jose María Tenias Burillo for providing us with his methodological support. This work was funded by a grant (FISCAM AN-2008/21) awarded to Alfredo J. Lucendo.

Conflict of interests None.

\section{References}

1. Furuta GT, Liacouras CA, Collins MH, et al. Eosinophilic esophagitis in children and adults: a systematic review and consensus recommendations for diagnosis and treatment. Gastroenterology. 2007;133:1342-1363.

2. Lucendo AJ, Lucendo B. An update on the immunopathogenesis of eosinophilic esophagitis. Expert Rev Gastroenterol Hepatol. 2010;4:141-148.

3. Trier JS. Celiac sprue. N Engl J Med. 1991;325:1709-1719.

4. Marsh MN. Gluten, major histocompatibility complex, and the small intestine. A molecular and immunobiologic approach to the spectrum of gluten sensitivity ('celiac sprue'). Gastroenterology. 1992;102:330-354.

5. Rewers M. Epidemiology of celiac disease: what are the prevalence, incidence, and progression of celiac disease? Gastroenterology. 2005; 128:S47-S51.

6. Thorsby E, Lie BA. HLA associated genetic predisposition to autoimmune diseases: genes involved and possible mechanisms. Transpl Immunol. 2005;14:175-182. 
7. Sollid LM, Markussen G, Ek J, et al. Evidence for a primary association of celiac disease to a particular HLA-DQ alpha/beta heterodimer. J Exp Med. 1989;169:345-350.

8. Kagalwalla AF, Shah A, Ritz S, et al. Cow's milk proteininduced eosinophilic esophagitis in a child with gluten-sensitive enteropathy. J Pediatr Gastroenterol Nutr. 2007;44:386-388.

9. Verzegnassi F, Bua J, De Angelis P, et al. Eosinophilic oesophagitis and coeliac disease: is it just a casual association? Gut. 2007;56:1029-1030.

10. Quaglietta L, Coccorullo P, Miele E, et al. Eosinophilic oesophagitis and coeliac disease: is there an association? Aliment Pharmacol Ther. 2007;26:487-493.

11. Leslie C, Mews C, Charles A, et al. Celiac disease and eosinophilic esophagitis: a true association. J Pediatr Gastroenterol Nutr. 2010;50:397-399.

12. Lipschitz B. Eosinophilic oesophagitis: association with HLADQ2 and HLA-DQ8. Gastroenterology. 2007;132:A282.

13. Barker JM, Liu E. Celiac disease: pathophysiology, clinical manifestations and associated autoimmune conditions. $A d v$ Pediatr. 2008;55:349-365.

14. Green PH, Jabri B. Coeliac disease. Lancet. 2003;362:383-391.

15. Rodrigo L. Celiac disease. World J Gastroenterol. 2006;12: $6585-6593$
16. Martín-Muñoz MF, Lucendo AJ, Navarro M, et al. Food allergy and eosinophilic esophagitis: two cases studies. Digestion. 2006;74: $49-54$.

17. Straumann A, Simon HU. Eosinophilic esophagitis: escalating epidemiology? J Allergy Clin Inmunol. 2005;115:418-419.

18. Patel SM, Falchuk KR. Three brothers with dysphagia caused by eosinophilic esophagitis. Gastrointest Endosc. 2005;61:165-167.

19. Weller PF. The immunobiology of eosinophils. N Engl J Med. 1991;324:1110-1118.

20. Collins MH, Blanchard C, Albonia JP, et al. Clinical, pathologic, and molecular characterization of familial eosinophilic esophagitis compared with sporadic cases. Ciln Gastroenterol Hepatol. 2008;6:621-629.

21. Kapel RC, Miller JK, Torres C, et al. Eosinophilic esophagitis: a prevalent disease in the United States that affects all age groups. Gastroenterology. 2008;134:1316-1321.

22. Katzka DA. Demographic data and symptoms of eosinophilic esophagitis in adults. Gastrointestinal Endosc Clin North Am. 2008;18:25-32.

23. Soma J, Brown-Whitehorn TA, Spergel JM. Association of eosinophilic gastrointestinal disorders with other atopic disorders. Immunol Allergy Clin N Am. 2009;29:85-97. 